\title{
Insatisfação com a imagem corporal no período pré-gestacional e fatores associados
}

\section{Body image dissatisfaction in pre-gestational period and associated factors Insatisfacción con la imagen corporal en el periodo pregestacional y factores asociados}

Adriana Passanha

Ana Paula Bortoletto Martins

Ana Carolina Feldenheimer da Silva

Maria Helena D’Aquino Benício

Faculdade de Saúde Pública da Universidade de São Paulo (FSP/USP)

\begin{abstract}
Resumo
Objetivo. Identificar fatores associados à insatisfação com a imagem corporal reportada por mulheres no período pré-gestacional. Métodos. Análise transversal de estudo de coorte de gestantes do Município de São Paulo. A percepção da imagem corporal foi avaliada pela Escala de Silhuetas. Foram obtidas características socioeconômicas, antropométricas, de estilo de vida e reprodutivas. A associação entre insatisfação com a imagem corporal e fatores de estudo foi analisada por modelos de regressão linear múltiplos hierárquicos. Resultados. A maioria das mulheres estava satisfeita com sua imagem corporal; porém, 36,7\% desejariam ser mais magras. Baixa escolaridade, prática de caminhada e aumento do Índice de Massa Corporal associaram-se à insatisfação com a imagem corporal, após ajuste para as variáveis incluídas no modelo múltiplo. Conclusões. Caracterizar diferentes determinantes da insatisfação com a imagem corporal é fundamental para formular intervenções no período pré-gestacional, visto que promover uma imagem corporal positiva nesse período contribuirá para gestações mais saudáveis.

Palavras-chave: Auto-imagem; Percepção; Imagem corporal; Mulheres; Gravidez.
\end{abstract}

\begin{abstract}
Objective. To identify associated factors with body image dissatisfaction reported by women in pre-gestational period. Methods. Cross-sectional analysis about a cohort study of pregnant women in São Paulo. The Scale of Silhouettes was employed to evaluate the perception of body image. Socioeconomic, anthropometric, lifestyle and reproductive characteristics were obtained. The association between body image dissatisfaction and study factors was evaluated by hierarchical multiple linear regression models. Results. Most of the women was satisfied with their body image, but $36.7 \%$ wanted to be thinner. Low education, walking practice and increasing on Body Mass Index were associated with body image dissatisfaction, after adjust variables included in the multiple model. Conclusions. To characterize different determinants of body image dissatisfaction is fundamental to formulate interventions in pre-gestational period, considering that promoting a positive body image in this period will contribute to healthier gestations.
\end{abstract}

Key-words: Self concept; Perception; Body image; Women; Pregnancy.

\section{Resumen}

Objetivo. Identificar factores asociados con la insatisfacción de la imagen corporal reportados por mujeres en el pre-gestacional. Métodos. Análisis transversal de estudio de cohorte de embarazadas en São Paulo. La percepción de la imagen corporal fue evaluada por la Escala de Siluetas. Obtuvimos datos socioeconómico, antropométricos estilo de vida y reproductiva. La asociación entre insatisfacción de la imagen corporal y factores de estudio fueron analizados por múltiples modelos de regresión lineal jerárquico. Resultados. La mayoría de las mujeres estaban satisfechas con su imagen, pero 36,7\% le gustaría ser más delgada. Educación bajo, caminar y mayor Índice de Masa Corporal se asociaron con la insatisfacción de la imagen, después de ajustar por variables de el modelo múltiple. Conclusiones. Caracterizar los determinantes de la insatisfacción con la imagen corporal es crucial para formular intervenciones en el pre-embarazo, ya que la promoción de una imagen corporal positiva en este período traerá embarazos saludables.

Palabras-clave: Autoimagen; Percepción; Imagen corporal; Mujeres; Embarazo.

\section{Introdução}

O termo "imagem corporal" refere-se à figura que

Endereço 1: Avenida Doutor Arnaldo, 715 - Cerqueira César, São Paulo, SP. CEP: 02146-904. Telefones: 3061-7701/3061-7705. Fax: 3061-7130. temos em mente a respeito do tamanho, estrutura e forma do nosso corpo, e aos sentimentos relacionados a essas características (Slade, 1994). A imagem corporal tem conceito multidimensional, envolvendo aspectos subjetivos e perceptivos. Estes últimos incluem a percepção atual e ideal de tamanho e forma corporal que o indivíduo possui de si mesmo (Slade, 
1994; Almeida, Santos, Pasian \& Loureiro, 2005; Duncombe, Wertheim, Skouteris, Paxton \& Kelly, 2008; Secchi, Camargo \& Bertoldo, 2009).

Quando há um distúrbio no componente perceptivo - alteração na forma como o indivíduo percebe o próprio corpo -, a pessoa passa a se ver de forma distorcida, o que caracteriza a "distorção da imagem corporal" (Slade, 1994; Sato, Timerman, Fabbri, Scagliusi \& Kotait, 2011). A "insatisfação com a imagem corporal" é um dos distúrbios perceptivos mais comuns, e reflete o desejo de ser diferente daquilo que se é. Tal insatisfação pode ser avaliada pela discrepância entre a percepção de como o indivíduo se vê - imagem percebida - e o desejo relacionado a um tamanho e formato de corpo - imagem desejada (Almeida et al., 2005; Sato et al., 2011; Chang, Chao \& Kenney, 2006).

$\mathrm{Na}$ década de 70, surgiu o ideal de corpo atlético e com formas definidas, fazendo referência ao "corpo magro" (Scagliusi \& Lourenço, 2011). Desde então, a magreza se tornou sinônimo do que é belo e cultuado, e o "corpo gordo" passou a ser o antiideal de beleza (Scagliusi \& Lourenço, 2011; Schwartz \& Brownell, 2004). As mulheres são particularmente afetadas por estas questões, e acabam por considerar o corpo magro como um sinônimo do que é (ou deve ser) normal (Almeida et al., 2005; Chang et al., 2006). Com os atuais padrões de beleza impostos pela mídia e pela pressão social, o corpo real e o corpo ideal entram em conflito, levando a distorções da imagem corporal e, por conseguinte, insatisfação com o tamanho e o formato corporais (Secchi et al., 2009; Boscaglia, Skouteris \& Wertheim, 2003; Loth, Bauer, Wall, Berge \& Neumark-Sztainer, 2011). Tal insatisfação pode acarretar práticas não-saudáveis de emagrecimento, o que podem levar ao desenvolvimento de transtornos alimentares - os quais vêm sendo observado com mais frequência atualmente -, causando importantes danos à saúde (Freitas, Novello, Gastaldob \& Justino, 2009).

Desde o início da gestação, a mulher sofre alterações físicas, hormonais e psicológicas, que podem resultar em mudanças significativas da percepção de sua imagem corporal (Duncombe et al., 2008; Chang et al., 2006; Loth et al., 2011; Skouteris, Carr, Wertheim, Paxton \& Duncombe, 2005). Essas mudanças ocorrem, inclusive, entre mulheres no período pré-gestacional que estão com o peso adequado (Fox \& Yamaguchi, 1997). Destacase ainda que mulheres insatisfeitas com seu corpo no início da gestação permanecem insatisfeitas ao longo de toda gravidez (Rauff \& Downs, 2011), o que pode acarretar consequências negativas à gestante e ao feto (Boscaglia et al., 2003; Skouteris et al., 2005).

A gravidez, para algumas mulheres, corresponde a um hiato temporário da relação existente entre magreza e feminilidade (Chang et al., 2006), devido à imposição de que a mulher precisa ter um corpo magro para se enquadrar aos padrões de beleza socialmente estabelecidos (Almeida et al., 2005; Chang et al., 2006; Scagliusi \& Lourenço, 2011; Schwartz \& Brownell, 2004). Compreender melhor o comportamento feminino ainda antes de as mulheres engravidarem oferece elementos protetores contra fatores relacionados à má saúde materna, como insatisfação com a imagem corporal durante a gestação, e práticas não-saudáveis de alimentação, inatividade física ao longo da gravidez e no pós-parto (Skouteris et al., 2005; Rauff \& Downs, 2011; Downs, DiNallo \& Kirner, 2008). Sendo assim, o objetivo do presente estudo é identificar fatores associados à insatisfação com a imagem corporal reportada por gestantes no período pré-gestacional.

\section{Métodos}

O presente estudo apresenta análise transversal de dados de um estudo de intervenção do tipo coortecontrolado de gestantes que realizaram pré-natal em cinco unidades básicas de saúde do Município de São Paulo, SP. Foram utilizadas informações de período prévio à intervenção. Os critérios de inclusão foram: mulheres saudáveis com gestação de baixo risco, idade superior a 18 anos e idade gestacional igual ou inferior a 16 semanas no momento da primeira entrevista. As gestantes da coorte "controle" receberam orientações apenas do serviço público de pré-natal. Na coorte "intervenção" as gestantes receberam orientações sobre alimentação saudável e estímulo à prática de caminhada por nutricionista da equipe de pesquisa não envolvida na coleta de dados, nas consultas de pré-natal. Na coorte controle, foram captadas 225 gestantes entre abril e junho de 2005 e na coorte intervenção, que começou onze meses após o início da primeira coorte, 160 mulheres iniciaram o seguimento. Destaca-se que as gestantes das duas coortes foram semelhantes com relação a variáveis socioeconômicas, história reprodutiva e estilo de vida (dados não apresentados).

Para avaliar a percepção da imagem corporal, utilizou-se a Escala de Silhuetas (Figure Rating Scale) proposta por Sorensen e Stunkard (1993). Este instrumento corresponde à avaliação perceptual (relacionado à precisão da percepção do tamanho e da forma corporal) e é composto por nove figuras de mulheres não-gestantes, cada uma com um número correspondente (o número 1 refere-se ao desenho de uma mulher com baixo peso e o número 9 de uma mulher com obesidade grave). $\mathrm{Na}$ entrevista com as gestantes, foram realizadas duas perguntas referentes à percepção da imagem corporal em momento anterior à gestação: "1. Escolha a figura que melhor descreve a senhora antes da gravidez", e "2. Qual destes desenhos representa a aparência que a senhora gostaria de ter antes da gestação?’. Assim, a primeira pergunta corresponde à imagem percebida, 
e a segunda à imagem desejada. A escala, referente ao período pré-gestacional, foi aplicada entre a $26^{\mathrm{a}}$ e a $29^{\mathrm{a}}$ semana de gestação.

A insatisfação com a imagem corporal foi obtida calculando-se a diferença entre o valor da figura que representa a aparência percebida e o valor da que representa a aparência desejada (Skouteris et al., 2005). Quanto maior é o valor resultante, mais magra a mulher gostaria de ser. Para valores negativos, o desejo é de ter o peso maior do que o apresentado.

Como fatores associados, foram estudadas variáveis referentes a: renda mensal do domicílio (em salários mínimos) - com base os valores do ano 2005 (Tribunal Regional do Trabalho da $3^{\mathrm{a}}$ Região, 2012); escolaridade (em anos de estudo completos); mora com companheiro (sim ou não); prática de caminhada ( $\operatorname{sim}$ ou não); paridade (número de gestações); e estado nutricional inicial (pelo cálculo do Índice de Massa Corporal $-\mathrm{IMC}=$ peso/estatura ${ }^{2}$ ) - o qual foi avaliado segundo parâmetros da Organização Mundial da Saúde (WHO, 1995). Estas informações foram obtidas na primeira entrevista com as gestantes (idade gestacional média de 12 semanas), em momento anterior à aplicação da intervenção.

Para análise descritiva, foram calculadas frequências e médias das variáveis estudadas e respectivos intervalos com $95 \%$ de confiança (IC Figura 1

Modelo hierárquico de organização das variáveis estudadas.

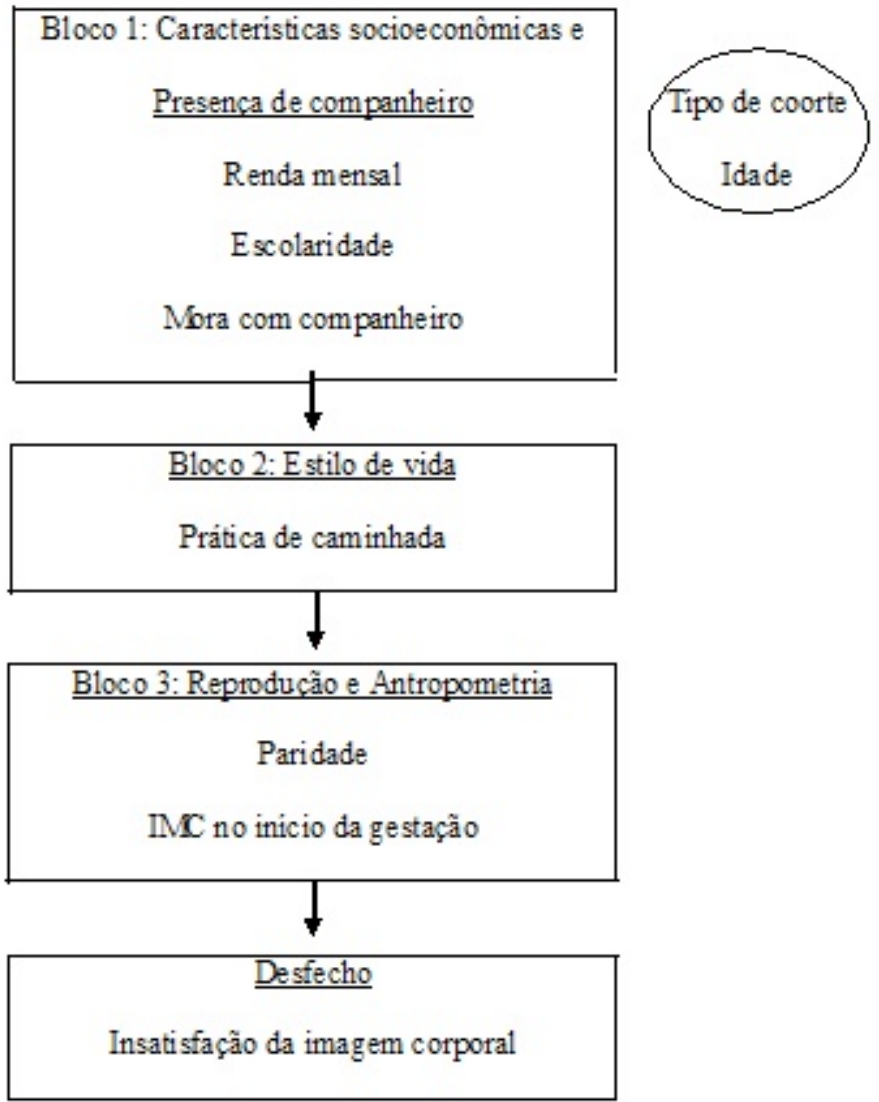

95\%). Calculou-se a média e IC 95\% da insatisfação corporal para cada fator estudado, ajustadando-se para tipo de coorte e idade.

Para verificar a associação entre satisfação com a imagem corporal e fatores de estudo, foram utilizados modelos de regressão linear múltiplos (Kleinbaum, Kupper, Muller \& Nizam, 1997). Inicialmente, foram realizadas análises de regressão linear simples para cada variável independente e a variável resposta (insatisfação com a imagem corporal). As variáveis que apresentaram $\mathrm{p}<0,20$ na análise bivariada foram incluídas no modelo múltiplo.

Para inclusão das variáveis individuais no modelo múltiplo, utilizou-se a estratégia de modelagem hierarquizada. Nessa estratégia, a hierarquização das variáveis independentes é estabelecida a partir de um marco conceitual, sendo divididas em blocos hierárquicos (Victora, Huttly, Fuchs \& Olinto, 1997). A Figura 1 apresenta o modelo hierárquico das variáveis de estudo, agrupadas em três blocos: 1) características socioeconômicas e presença de companheiro, 2) estilo de vida, e 3) características reprodutiva e antropométrica (Figura 1).

As variáveis do bloco 1 foram introduzidas na primeira etapa da modelagem, sendo ajustadas pelo tipo de coorte e idade. $\mathrm{Na}$ etapa seguinte, foi incluída a variável referente ao bloco 2 (prática de caminhada), 
Figura 2

Proporção (\%) de mulheres insatisfeitas e satisfeitas com a imagem corporal segundo classificação do IMC. Município de São Paulo, 2005.

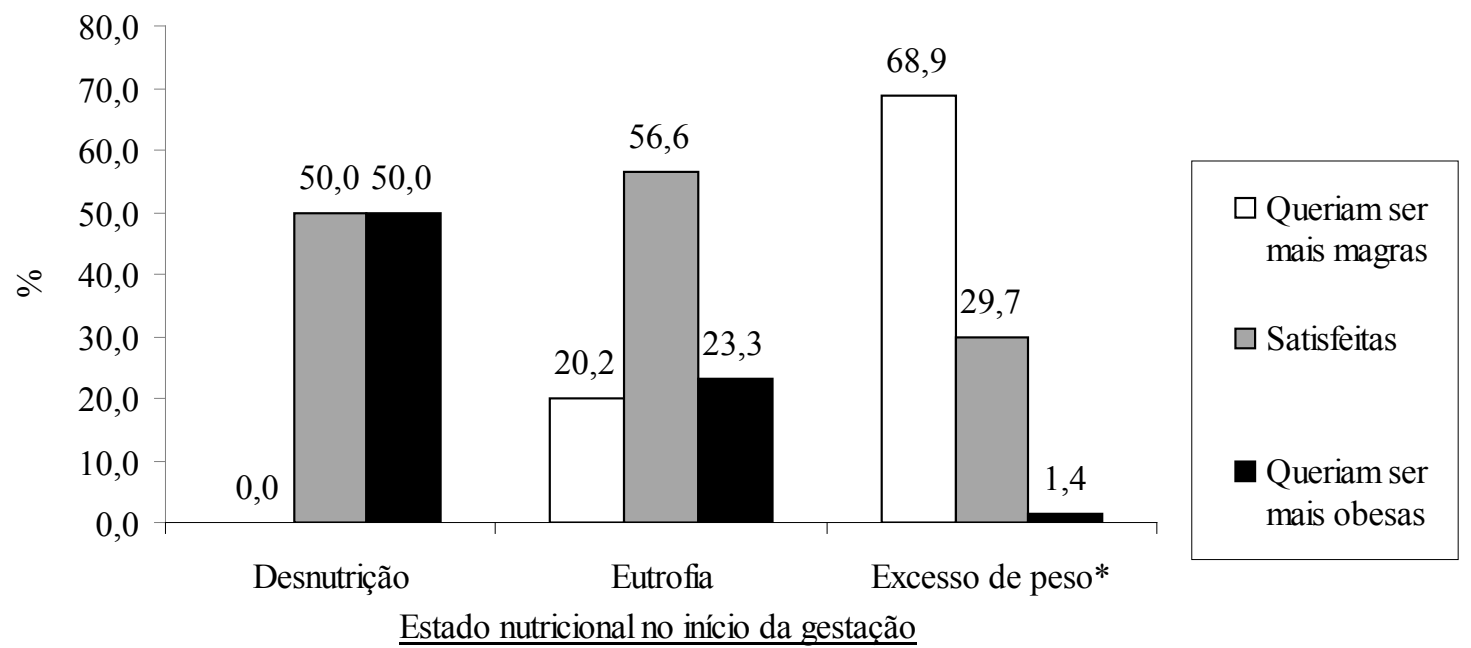

*excesso de peso: sobrepeso + obesidade

e, por fim, houve a inclusão das variáveis do bloco 3. Para considerar associação estatisticamente significativa adotou-se nível de significância igual a 0,05 . O software Stata 10.1 foi utilizado em todas as etapas de análises.

O projeto de pesquisa foi aprovado pelo Comitê de Ética da Faculdade de Saúde Pública da Universidade de São Paulo. Todas as gestantes entrevistadas assinaram o Termo de Consentimento Livre e Esclarecido após serem informadas sobre a pesquisa.

\section{Resultados}

Das 385 mulheres captadas no início do estudo, 330 foram consideradas elegíveis para participar do mesmo. No presente estudo foram efetivamente estudadas 237 gestantes, sendo que as perdas de seguimento representaram $28,2 \%$ do total de mulheres captadas consideradas elegíveis. Não foram verificadas diferenças significativas das características socioeconômicas entre as gestantes estudadas e as perdas (dados não apresentados).

A idade das participantes variou de 18 a 42 anos, sendo a média igual a 26,1 anos $(\mathrm{DP}=5,35)$. A maioria delas encontrava-se no estrato de renda intermediário, morava com companheiro e era multípara. A média de escolaridade foi de 8,6 anos de estudo ( $\mathrm{DP}=0,19)$. Em relação ao IMC, houve maior prevalência de mulheres eutróficas, seguidas por aquelas com sobrepeso; a média de IMC foi de 24,32 (DP=0,30) (Tabela 1).

A maioria da população estudada $(47,1 \%)$ estava satisfeita com sua imagem corporal; porém, 36,7\% queriam ser mais magras. A percepção da imagem corporal foi distinta segundo o estado nutricional das gestantes: dentre as mulheres com baixo peso, $50,0 \%$ estavam satisfeitas com sua imagem corporal e nenhuma gostaria de ser mais magra; dentre as eutróficas, 20,2\% gostaria de ser mais magra; já aquelas com excesso de peso (sobrepeso ou obesidade), $68,9 \%$ expressaram que queriam ser mais magras, e apenas 29,7\% estavam satisfeitas com a própria imagem (Figura 2) (inserir aqui a Figura 2).

Na Tabela 2, observa-se que a média de insatisfação corporal tendeu a aumentar com o aumento da renda, e diminuir com o aumento da escolaridade. As mulheres que moram com companheiro, as que não caminham e as primíparas tendem a ser mais satisfeitas com sua imagem corporal. No entanto, o aumento da insatisfação com a imagem corporal associou-se significativamente somente com o aumento do IMC (inserir aqui a Tabela 2).

$\mathrm{Na}$ análise de regressão linear simples, as variáveis referentes à renda mensal e presença de companheiro não alcançaram significância estatística; as demais entraram para o modelo múltiplo (Tabela 3) (inserir aqui a Tabela 3 ). Neste modelo, observouse que a escolaridade foi inversamente associada com a insatisfação da imagem corporal $(p=0,032)$. No segundo bloco do modelo, verificou-se que as mulheres que caminham são mais insatisfeitas com sua imagem ( $\mathrm{p}=0,037)$, após controle por escolaridade. O IMC manteve-se associado positivamente com o desfecho, após ajuste pelas variáveis anteriores $(\mathrm{p}<0,001)$.

\section{Discussão}

No presente estudo, observou-se que cerca de um terço $(36,7 \%)$ das mulheres gostaria de ser mais magra. Os fatores associados significativamente à insatisfação corporal no período pré-gestacional foram baixa escolaridade, prática de caminhada e aumento de IMC.

Em estudo longitudinal realizado na Austrália, 
Tabela 1

Distribuição (n) e proporção (\%) de características socioeconômicas, de estilo de vida e de estado nutricional das gestantes. Município de São Paulo, 2005.

\begin{tabular}{|c|c|c|}
\hline Variável & $n$ & $\%$ \\
\hline \multicolumn{3}{|l|}{ Tipo de coorte } \\
\hline Controle & 131 & 55,2 \\
\hline Intervenção & 106 & 44,7 \\
\hline \multicolumn{3}{|l|}{ Faixa etária } \\
\hline $18 \mid-25$ anos & 105 & 44,3 \\
\hline $25 \mid-30$ anos & 62 & 26,2 \\
\hline $30 \mid-35$ anos & 56 & 23,6 \\
\hline 35 anos e mais & 14 & 5,9 \\
\hline \multicolumn{3}{|l|}{ Renda mensal (em salários mínimos)\$ } \\
\hline$<2$ salários mínimos & 64 & 29,6 \\
\hline $2 \mid-5$ salários mínimos & 110 & 50,9 \\
\hline 5 salários mínimos e mais & 42 & 19,4 \\
\hline \multicolumn{3}{|l|}{ Escolaridade (anos de estudo) } \\
\hline $0 \mid-8$ anos & 73 & 32,3 \\
\hline $8 \mid-11$ anos & 69 & 30,5 \\
\hline 11 anos e mais & 84 & 37,2 \\
\hline \multicolumn{3}{|l|}{ Mora com companheiro } \\
\hline $\operatorname{Sim}$ & 178 & 75,4 \\
\hline Não & 58 & 24,6 \\
\hline \multicolumn{3}{|l|}{ Prática de caminhada no lazer } \\
\hline Caminha & 214 & 90,3 \\
\hline Não caminha & 23 & 9,7 \\
\hline \multicolumn{3}{|l|}{ Paridade } \\
\hline Primípara & 98 & 41,7 \\
\hline Multípara & 137 & 58,3 \\
\hline \multicolumn{3}{|l|}{ Estado nutricional inicial } \\
\hline Baixo peso (IMC < 18,5kg/m2) & 7 & 3,0 \\
\hline Eutrofia (IMC 18,5 -| 25,0 kg/m2) & 147 & 62,2 \\
\hline Sobrepeso (IMC 25,0 -| 30,0 kg/m2) & 63 & 26,7 \\
\hline Obesidade (IMC > 30,0 kg/m2) & 19 & 8,1 \\
\hline
\end{tabular}

observou-se que $76,6 \%$ das mulheres estudadas no período pré-gestacional queriam ser mais magras; as demais estavam satisfeitas com seus corpos (Skouteris et al., 2005). Outro estudo conduzido no mesmo país constatou que, no período anterior à gestação, $47,2 \%$ da população estudada queria ser mais magra, e apenas $5,7 \%$ desejou ter um peso maior (Duncombe et al., 2008).

Dados do VIGITEL (Vigilância de Doenças Crônicas por Inquérito Telefônico) 2006 mostram que a prevalência de excesso de peso (IMC $\geq 25 \mathrm{~kg}$ / $\mathrm{m}^{2}$ ) entre mulheres adultas da cidade de São Paulo foi de 42,8\% (Ministério da Saúde, 2006). No presente estudo, esta prevalência foi de $34,8 \%$. Considerando que o inquérito incluiu mulheres com idade igual ou maior a 18 anos e que as prevalências de excesso de peso foram maiores nas faixas etárias mais elevadas - acima de 45 anos (Ministério da Saúde, 2006). É provável que os resultados encontrados neste estudo sejam semelhantes aos do VIGITEL.

Corroborando os resultados do presente estudo, outras pesquisas realizadas com mulheres não grávidas saudáveis encontraram a associação entre elevação do IMC e o aumento da insatisfação com a imagem corporal. Estudo realizado nos EUA concluiu que as mulheres com peso mais elevado estavam mais insatisfeitas com sua imagem corporal (DiPietro, Millet, Costigan, Gurewitsch \& Caulfield, 2003). Em pesquisa observacional com mulheres brasileiras de idade semelhante à deste estudo, foi constatado que quanto maior o IMC, maior foi a percepção de que o próprio corpo está acima do peso considerado "ideal" e maior foi a insatisfação com o próprio corpo (Secchi et al., 2009). Estudo de revisão aponta que mulheres com sobrepeso e obesidade são mais insatisfeitas com seus corpos do que eutróficas (Schwartz \& Brownell, 2004). De acordo com os autores, isso ocorre provavelmente porque mulheres com excesso de peso são estigmatizadas pela sociedade por não conseguirem manter o controle sobre seus corpos, o que as transforma em fracas, preguiçosas, sem força de vontade, e estereótipos do gênero. Considerando 
Tabela 2

Média da insatisfação com a imagem corporal segundo características das gestantes (ajustada por tipo de coorte e idade). Município de São Paulo, 2005.

\begin{tabular}{lcc}
\multicolumn{1}{c}{ Variável } & Média & IC 95\% \\
\hline Renda mensal (em salários mínimos) & 0,29 & 0,$05 ; 0,53$ \\
$<2$ salários mínimos & 0,34 & 0,$19 ; 0,49$ \\
2 |- 5 salários mínimos & 0,39 & 0,$12 ; 0,67$ \\
5 salários mínimos e mais & & \\
Escolaridade (anos de estudo) & 0,52 & 0,$27 ; 0,76$ \\
$0 \mid-8$ anos & 0,38 & 0,$23 ; 0,52$ \\
$8 \mid-11$ anos & 0,23 & 0,$01 ; 0,45$ \\
11 anos e mais & 0,30 & 0,$14 ; 0,46$ \\
Mora com companheiro & 0,50 & 0,$21 ; 0,79$ \\
Sim & & \\
Não & 0,39 & 0,$24 ; 0,53$ \\
Prática de caminhada no lazer & $-0,07$ & $-0,54 ; 0,39$ \\
Caminha & & \\
Não caminha & 0,20 & $-0,03 ; 0,43$ \\
Paridade & 0,44 & 0,$25 ; 0,64$ \\
Primípara & & $-1,10 ;-0,55$ \\
Multípara & $-0,83$ & $-0,13 ; 0,15$ \\
Estado nutricional inicial & 0,01 & 0,$68 ; 1,00$ \\
Baixo peso (IMC $<18,5 \mathrm{~kg} / \mathrm{m}^{2}$ ) & 0,84 & 1,$37 ; 1,98$ \\
Eutrofia (IMC $\left.18,5-\mid 25,0 \mathrm{~kg} / \mathrm{m}^{2}\right)$ & 1,68 & \\
Sobrepeso (IMC $\left.25,0-\mid 30,0 \mathrm{~kg} / \mathrm{m}^{2}\right)$ & &
\end{tabular}

que as prevalências de excesso de peso vêm aumentando no mundo todo (WHO, 2000), é preciso atentar-se ao fato de que pessoas com excesso de peso e que desejam diminuir seu peso podem desenvolver transtornos alimentares, pelo fato de realizarem dietas restritivas e inadequadas (Freitas et al., 2009; Petribu et al., 2006).

Quanto à associação da insatisfação com a imagem corporal e os anos de estudo, achados semelhantes foram encontrados em uma coorte na qual as gestantes que possuíam menor escolaridade apresentaram-se mais insatisfeitas com sua imagem no período pré-gestacional (Herring et al., 2008). Esse resultado pode ser atribuído ao fato de que mulheres com maior escolaridade têm mais esclarecimento sobre como estão inseridas na sociedade, e se vêem de forma mais positiva, inclusive em relação à imagem corporal.

Em relação à prática de atividade física, outros autores constataram que mulheres fisicamente ativas apresentaram-se mais satisfeitas com sua imagem corporal no período pré-gestacional (Boscaglia et al., 2003), diferentemente do achado deste estudo. Uma explicação para este resultado pode ser o efeito de causalidade reversa, isto é, as mulheres insatisfeitas com sua imagem corporal passam a caminhar para reverter esse quadro. Entretanto, pode-se afirmar que, se as mulheres praticarem atividade física mesmo antes de engravidarem, possivelmente terão melhor percepção de sua imagem corporal e é mais provável que mantenham boa saúde fisiológica durante a gestação (Downs et al., 2008).

Em relação à paridade, os resultados encontrados na literatura são bastante divergentes. Um estudo não encontrou relação entre insatisfação com a imagem corporal e paridade (Herring et al., 2008), outro observou que as primíparas apresentam-se mais satisfeitas com sua imagem corporal do que as multíparas (DiPietro et al., 2003), e um terceiro constatou que, entre mulheres inglesas, primíparas têm maior preocupação com sua imagem corporal do que multíparas (Fox \& Yamaguchi, 1997). No presente estudo, foi observado que, na análise bivariada (controlada por tipo de coorte e idade) as multíparas se apresentaram mais insatisfeitas do que as primíparas, mas a variável paridade perdeu significância na análise múltipla. Possivelmente, a associação observada na análise bruta também pode ser explicada pelo IMC: dentre as multíparas, $45,6 \%$ estavam com excesso de peso, enquanto $18,7 \%$ das primíparas pertenciam à mesma categoria de IMC. Os maiores valores de IMC encontrados entre as multíparas podem ser explicados pela associação entre paridade e retenção de peso pós-parto, visto que tal retenção pode levar essas mulheres ao excesso de peso após a gestação (Lacerda \& Leal, 2004).

Não foi observada associação entre renda mensal ou presença de companheiro e insatisfação com a imagem corporal na análise bruta. Corroborando esses achados, outros estudos também não encontraram tal associação (Skouteris et al., 2005; DiPietro et al., 2003; Herring et al., 2008). Alguns 
Tabela 3

Modelos de regressão linear bruto e múltiplo da insatisfação com a imagem corporal no periodo prégestacional e fatores associados. Município de São Paulo, 2005.

\begin{tabular}{|c|c|c|c|c|c|c|}
\hline & \multicolumn{3}{|c|}{ Bruto $^{s}$} & \multicolumn{3}{|c|}{ Múltiplo } \\
\hline Variável & $\beta$ & $\mathrm{IC} 95 \%$ & $\mathrm{p}$ & $\bar{\beta}$ & $\mathrm{IC} 95 \%$ & $\mathrm{p}$ \\
\hline Bloco 1 & & & & & & \\
\hline Renda mensal ${ }^{\pi}$ & 0,00 & $-0,00 ; 0,00$ & 0,693 & - & - & - \\
\hline Escolaridade & $-0,06$ & $-0,11 ;-0,01$ & 0,032 & $-0,06$ & $-0,11 ;-0,01$ & 0,032 \\
\hline Mora com companheiro & $-0,20$ & $-0,54 ; 0,13$ & 0,235 & - & - & - \\
\hline Bloco 2 & & & & & & \\
\hline $\begin{array}{l}\text { Prática de caminhada no } \\
\text { lazer }^{\dagger}\end{array}$ & 0,46 & $-0,03 ; 0,95$ & 0,065 & 0,55 & 0,$03 ; 1,07$ & 0,037 \\
\hline Bloco 3 & & & & & & \\
\hline Paridade & 0,24 & $-0,07 ; 0,56$ & 0,133 & 0,01 & $-0,13 ; 0,16$ & 0,840 \\
\hline IMC & 0,13 & 0,$11 ; 0,16$ & $<0,001$ & 0,13 & 0,$10 ; 0,16$ & $<0,001$ \\
\hline
\end{tabular}

${ }_{\S}$ Modelo Bruto: ajustado por tipo de coorte e idade.

"Variáveis incluídas no modelo no formato contínuo.

†Variáveis incluídas no modelo no formato dicotômico.

Bloco 2: ajustado pelo Modelo Bruto e pelo Bloco 1.

Bloco 3: ajustado pelo Modelo Bruto, pelo Bloco 1 e pelo Bloco 2.

autores sugerem que esse resultado pode ser atribuído à falta de heterogeneidade das populações estudadas em relação a essas características, sendo interessante estudar mulheres de diferentes estratos econômicos e estados civis para verificar essa associação (Skouteris et al., 2005; DiPietro et al., 2003).Devido aos resultados positivos encontrados entre aumento do IMC e insatisfação com a imagem corporal, foram realizadas as mesmas análises tendo a variável IMC como desfecho (dados não apresentados). Alguns dos achados foram bastante similares: as variáveis escolaridade e prática de caminhada mantiveramse associadas ao IMC da mesma forma que se associaram à insatisfação da imagem corporal. Já a paridade apresentou associação significativa com o IMC, mesmo com todos os ajustes da análise múltipla.

Diversos estudos que encontraram a satisfação com a imagem corporal ao longo da gestação consideram que as gestantes se adaptam rapidamente às alterações corporais decorrentes da gravidez porque tais mudanças correspondem a uma gestação completa e bem-sucedida (Duncombe et al., 2008; Boscaglia et al., 2003; Loth et al., 2011; Skouteris et al., 2005; Fox \& Yamaguchi, 1997). Corroborando tais achados, alguns autores supõem que a maior satisfação com a imagem corporal durante a gravidez também pode ser decorrente de as gestantes possuírem um novo papel social, e por isso estão mais protegidas da pressão de alcançar o corpo ideal (Loth et al., 2011). Entretanto, é provável que os sintomas que podem ocorrer na gestação (enjoos, náuseas, fadiga, dores de cabeça, etc.) tenham algum impacto na percepção da imagem corporal, principalmente no início da gravidez (Duncombe et al., 2008; Rauff \& Downs, 2011).
Ademais, as mulheres nas primeiras semanas de gestação estão mais vulneráveis a essa pressão, pois, muitas vezes, comparam suas mudanças corporais às de mulheres que ganham peso mas não estão grávidas, e assim, podem se perceber mais obesas do que estão (Duncombe et al., 2008).

De modo geral, as participantes deste estudo foram capazes de diferenciar os tamanhos e formas corporais percebidos e idealizados, visto que as mulheres com menor valor de IMC referiram sua imagem percebida às silhuetas correspondentes a mulheres mais magras, enquanto aquelas com IMC mais elevado referiram tal imagem às figuras de mulheres mais obesas. Assim como em outros estudos (Almeida et al., 2005; Sorensen \& Stunkard, 1993; Scagliusi et al., 2006), o uso da Escala de Silhuetas foi adequado para avaliar a percepção da imagem corporal, dada sua correspondência com o IMC. Ademais, é considerado fácil de ser aplicado, com potencial para avaliar a percepção da imagem corporal (Almeida et al., 2005), e consiste em um instrumento válido para avaliação da imagem corporal no Brasil (Scagliusi et al., 2006). Ainda, é importante observar que, no presente estudo, a maioria das mulheres apresentou boa escolaridade, e devido a isso, possivelmente as informações fornecidas pela população estejam bem acuradas (Buka, Goldstein, Spartos \& Tsuang, 2004).

Dentre as limitações, encontra-se a coleta retrospectiva de dados referentes à insatisfação com imagem corporal. Tal limitação também foi apontada por outros autores que procederam da mesma forma (Duncombe et al., 2008; Loth et al., 2011; Skouteris et al., 2005; Herring et al., 2008). Especificamente em pesquisas com gestantes, as mulheres podem 
perceber sua imagem corporal pré-gestacional de forma diferente quando estão grávidas, idealizando sua imagem nesse período, ou com a percepção de que sua imagem é mais negativa do que realmente é (Duncombe et al., 2008; Loth et al., 2011; Skouteris et al., 2005). A coleta retrospectiva de dados pode, ainda, ser afetada pelo viés de memória. Entretanto, deve-se considerar que é muito difícil e oneroso recrutar uma grande amostra de mulheres que não estão grávidas e acompanhá-las prospectivamente até engravidarem (Duncombe et al., 2008; Skouteris et al., 2005; Herring et al., 2008).

Outra possível limitação do presente estudo é a junção das mulheres pertencentes às coortes controle e intervenção na análise de dados. No entanto, todas as informações estudadas foram coletadas ainda no início da gestação ou de forma retrospectiva, em momento que a intervenção não havia sido aplicada. Ademais, a variável tipo de coorte foi incluída como ajuste nas análises de regressão linear bruta e múltipla.

A aceitação da imagem corporal, considerando a diversidade de tamanhos e formas de corpos, é bastante desafiadora. Cabe também aos profissionais de saúde trabalhar a questão do que é adequado em relação a tamanhos e formatos corporais, e de não supervalorizar a aparência como indicativo de valor (Scagliusi \& Lourenço, 2011), principalmente considerando as reações por vezes conflitantes que as gestantes têm em relação aos seus corpos (Chang et al., 2006).

Promover uma imagem corporal positiva ao longo de toda a gestação, e principalmente no seu início, pode ter impacto positivo em sua qualidade de vida por contribuir com a saúde da gestante, levando-a a práticas saudáveis de alimentação e atividade física e prevenindo a insatisfação com sua imagem durante a gravidez (Skouteris et al., 2005; Rauff \& Downs, 2011).

\section{Conclusões}

Baixa escolaridade, prática de caminhada e aumento de IMC associaram-se significativamente à insatisfação com a imagem corporal no período prégestacional em gestantes do Município de São Paulo. A caracterização dos diferentes determinantes de tal insatisfação é fundamental para formulação de estudos de intervenção abordando o tema ainda neste período. Este é um dos poucos estudos existentes sobre fatores associados à insatisfação com a imagem corporal no período pré-gestacional. Outras pesquisas são necessárias para melhor compreensão de tais fatores e de outros que podem influenciar essa insatisfação, como estudos de abordagem qualitativa, que podem aprofundar os conhecimentos acerca do tema.

\section{Referências}

Almeida, G. A. N., Santos, J. E., Pasian, S. R., \& Loureiro, S. R. (2005). Percepção de tamanho e forma corporal de mulheres: estudo exploratório. Psicologia em Estudo, 10 (1), 27-35.

Boscaglia, N., Skouteris, H., \& Wertheim, E. H. (2003). Changes in body image satisfaction during pregnancy: A comparison of high exercising and low exercising women. The Australian \& New Zealand Journal of Obstetrics \& Gynaecology, 43 (1), 41-45.

Buka, S. L., Goldstein, J. M., Spartos, E., \& Tsuang, M. T. (2004). The retrospective measurement of prenatal and perinatal events: accuracy of maternal recall. Schizophrenia Research, 71 (2-3), 417-426.

Chang, S. R., Chao, Y. M., \& Kenney, N. J. (2006). I am a woman and I'm pregnant: body image of women in Taiwan during the third trimester of pregnancy. Birth, 33 (2), 147-153.

DiPietro, J. A., Millet, S., Costigan, K. A., Gurewitsch, E., \& Caulfield, L. E. (2003). Psychosocial influences on weight gain attitudes and behaviors during pregnancy. Journal of the American Dietetic Association, 103 (10), 1314-1319.

Downs, D. S., DiNallo, J. M., \& Kirner, T. L. (2008). Determinants of pregnancy and postpartum depression: prospective influences of depressive symptoms, body image satisfaction, and exercise behavior. Annals of Behavioural Medicine: a publication of the Society of Behavioural Medicine, 36 (1), 54-63.

Duncombe, D., Wertheim, E. H., Skouteris, H., Paxton, S. J., $\&$ Kelly, L. (2008). How well do women adapt to changes in their body size and shape across the course of pregnancy? Journal of Health Psychology, 13 (4), 503-515.

Fox, P., \& Yamaguchi, C. (1997). Body image change in pregnancy: a comparison of normal weight and overweight primigravidas. Birth, 24 (1), 35-40.

Freitas, A. R., Novello, D., Gastaldob, L. T., \& Justino, P. F. (2009). Insatisfação da imagem corporal, práticas alimentares e de emagrecimento em adolescentes do sexo feminino. Revista Brasileira de Nutrição Clínica, 24 (3), 166-173.

Herring, S. J., Oken, E., Haines, J., Rich-Edwards, J. W., Rifas-Shiman, S. L., Kleinman, K. P., \& Gillman, M. W. (2008, Dezembro 22). Misperceived pre-pregnancy body weight status predicts excessive gestational weight gain: findings from a US cohort study. BMC Pregnancy and Childbirth, 8 (54). Acessado de http://www.biomedcentral.com/1471-2393/8/54

Kleinbaum, D. G., Kupper, L. L., Muller, K. E., \& Nizam, A. (1997). Applied regression analysis and other multivariable methods, ( $3^{\mathrm{a}}$ ed.). Duxbury: Pacific Grove.

Lacerda, E. M. A., \& Leal, M. C. (2004). Fatores associados com a retenção e o ganho de peso pós-parto: uma revisão sistemática. Revista Brasileira de Epidemiologia, 7 (2), 187-200.

Loth, K. A., Bauer, K. W., Wall, M., Berge, J., \& NeumarkSztainer, D. (2011). Body satisfaction during pregnancy. Body Image, 8 (3), 297-300.

Ministério da Saúde - Secretaria de Vigilância em Saúde Secretaria de Gestão Estratégica e Participativa. (2007). Vigitel Brasil 2006: Vigilância de fatores de risco e proteção para doenças crônicas por inquérito telefônico - Estimativas sobre a frequência e distribuição sócio-demográfica de fatores de risco e proteção para doenças crônicas nas capitais dos 26 estados brasileiros e no Distrito Federal em 2006. Brasília: Ministério da Saúde.

Petribu, K., Ribeiro, E. S., de Oliveira, F. M. F., Braz, C. I. A., Gomes, M. L. M., de Araújo, D. E., de Almeida, N. C. N., de Albuquerque, P. C., \& Ferreira, M. N. L. (2006). Transtorno da compulsão alimentar periódica em uma população de obesos mórbidos candidatos a cirurgia bariátrica do Hospital Universitário Oswaldo Cruz, em Recife - PE. Arquivos Brasileiros de Endocrinologia \& Metabologia, 50 (5), 901-908.

Rauff, E. L., \& Downs DS. (2011). Mediating effects of body image satisfaction on exercise behavior, depressive symptoms, and gestational weight gain during pregnancy. Annals of Behavioural Medicine: a publication of the Society of Behavioural Medicine, 42 (3), 381-390.

Sato, P. M., Timerman, F., Fabbri, A. D., Scagliusi, F. B., \& Kotait, M. S. (2011). A imagem corporal nos transtornos alimentares: como o terapeuta nutricional pode contribuir para 
o tratamento. In S. T. Philippi, M. Alvarenga, \& F. B. Scagliusi (Eds.), Nutrição e transtornos alimentares: avaliação e tratamento ( $1^{\mathrm{a}}$ ed., pp.475-495). Barueri: Editora Manole.

Scagliusi, F. B., Alvarenga, M., Polacow, V. O., Cordás, T. A., Queiroz, G. K. O., Coelho, D., Philippi, S. T., \& Lancha-Junior, A. H. (2006). Concurrent and discriminant validity of the Stunkard's figure rating scale adapted into Portuguese. Appetite, 47 (1), 77-82.

Scagliusi, F. B., \& Lourenço, B. H. (2011). A ditadura da beleza e suas consequências no discurso nutricional. In: S. T. Philippi, M. Alvarenga, \& F. B. Scagliusi. Nutrição e transtornos alimentares: avaliação e tratamento (1 $1^{\mathrm{a}}$ ed., pp.59-83). Barueri: Editora Manole.

Schwartz, M. B., \& Brownell, K. D. (2004). Obesity and body image. Body Image, 1 (1), 43-56.

Secchi, K., Camargo, B. V., \& Bertoldo, R. B. (2009). Percepção da imagem corporal e representações sociais do corpo. Psicologia: Teoria e Pesquisa, 25 (2), 229-236.

Skouteris, H., Carr, R., Wertheim, E. H., Paxton, S. J., \& Duncombe, D. (2005). A prospective study of factors that lead to body dissatisfaction during pregnancy. Body Image, 2 (4), 347-361.

Slade, P. D. (1994). What is body image? Behaviour Research and Therapy, 32 (5), 497-502.

Sorensen, T. I. A., \& Stunkard, A. J. (1993). Does obesity run in families because of genes? An adoption study using silhouettes as a measure of obesity. Acta Psychiatrica Scandinavica. Supplementum, 370, 67-72.

Tribunal Regional do Trabalho da $3^{\text {a }}$ Região. (2012). Evolução do salário mínimo. Acessado de http://www.trt3.jus.br/informe/ calculos/minimo.htm

Victora, C. G., Huttly, S. R., Fuchs, S. C., \& Olinto, M. T. A. (1997). The role of conceptual frameworks in epidemiological analysis: a hierarchical approach. International Journal of Epidemiology, 26 (1), 224-227.

World Health Organization. (1995). Physical Status: The Use and Interpretation of Anthropometry. (Technical Report Series, $\mathrm{n}^{\circ}$ 854: Report of a WHO Expert Committee). Geneva: WHO.

World Health Organization. (2006). Obesity: preventing and managing the global epidemic. (Technical Report Series, $\mathrm{n}^{\circ}$ 854: Report of a WHO Consultation). Geneva: WHO. 
Sobre os autores:

Adriana Passanha - Mestre em Ciências pela Faculdade de Saúde Pública da Universidade de São Paulo (FSP/USP).

E-mail: adriana.passanha@gmail.com

Ana Paula Bortoletto Martins - Doutoranda em Ciências pela Faculdade de Saúde Pública da Universidade de São Paulo (FSP/USP).

Ana Carolina Feldenheimer da Silva - Doutoranda em Ciências pela Faculdade de Saúde Pública da Universidade de São Paulo (FSP/USP).

Maria Helena D’Aquino Benício - Professora Associada do Departamento de Nutrição, Faculdade de Saúde Pública da Universidade de São Paulo (FSP/USP).

Fonte de financiamento: CNPq (Processo no. 505912/2004-0).

Conflito de interesses: os autores declaram não haver qualquer conflito de interesses.

Agradecimentos

Ao CNPq pelo financiamento e a todos que contribuíram para realização desse estudo, em especial à Silvia Cristina Konno, cuja participação foi fundamental na concepção e realização do estudo. 